\title{
Importance of Modeling and Simulation of Materials in Research
}

\begin{abstract}
All researchers have experience with various complicated phenomena and processes in materials and have contact with understanding of different complex dynamical systems. Consequently, one has to predict and optimize the system under study and compare the output results to its experimental setup. The experimental setup is done to confirm and spread out information about the system; however still, many researchers are not familiar with the results arising from the experimental setup or fabrications processes. To obtain valid experimental results, it is necessary to pay carful attentions to the tools, devices and the applied techniques for measuring and observations detectors. This experimentations and fabrications process cover all area from forming and characterizations of nanostructures to macrostructures. In system characterizations, it is common to apply system modeling to predict and represent the system by a mathematical model that is accomplishable due to the relations between the inputs and outputs defined by formulas.
\end{abstract}

In many cases, it is not practicable or/and possible to begin assembling the system setup due to cost effectiveness or/and environmental surrounding [1]. Therefore, the system setup can be realized by modeling and simulation and the researchers can run their systems to produce the phenomena that occurs in the actual setups. Modeling and simulation of materials is applied not only to verify and extend the experimental information, but also to explore and exploit it. There are many reasons why modeling and simulations of materials more important. Nowadays software utilities and hardware are powerful, flexible and low cost that modeling and simulations can be broadly used. The needs of modeling and simulations are increased so modeling and simulations are not restricted for system analysis, but also for e.g. designing $[2,3]$, optimizing $[4,5]$ and predicting the behavior of the outputs $[6,7]$. Another reason is the system under study can become larger than the actual experimental setup. For example, the low-cost fabrication of integrated micro-system and micro-sensors [8-10]. This industrial fabrication has high economic impact and for medical sensors have social impact [11]. The modeling and simulations of materials lead to a large change in multi-disciplinary applications and very important for those researchers whose research depends upon simulation.

Effective research outcome through simulation leads significant involvement of researchers in various aspects of modeling and simulation throughout the globe. As the simulation based research field is ever broadening, specialized journals required to disseminate those cutting-edge research outputs. However, in order to enable successful interdisciplinary interactions between different fields, the need for journals that cover all aspects of simulation based research is crucial. Moreover, with the rapidly growing research community, there is a necessity to provide free and easy access to high-quality research and innovations based on modeling and simulation. This can be achieved by introducing open access modeling and simulation journals. So, a new open access simulation journal by AIJR Publisher should be of interest to researchers working in the field of Modeling and Simulation. After long discussions with the editorial board members to choose the journal name in order to reflect the aims and scopes of the journal, we are proudly named it as "Journal of Modeling and Simulation of Materials" which reflects the importance of both system modeling and system simulation for prospective developments in all fields of theory and applications of materials. 
Journal of Modeling and Simulation of Materials (JMSM) is an international refereed journal dedicated to the latest advancements in modeling and simulation of materials. J. Mod. Sim. Mater. will provides a scholarly platform for research community in all aspects of modeling and simulation in chemistry, physics, material sciences, engineering and technology to publish the original full-length research papers, timely state-of-theart reviews, mini-review and short communications covering the fundamental and applied research. JMSM will follow rigorous peer review process as described elsewhere [12] to ensure quality of the journal. The presence of the first issue of the "Journal of Modeling and Simulation of Materials (JMSM)" provides the authors and readers opportunities of more information about the aim and scope of this journal. On behalf of the editorial board, I am inviting you to submit your original research articles to the Journal of Modeling and Simulation of Materials. I hope that with time Journal of Modeling and Simulation of Materials will become a premier publication for modeling and simulation in the modern researches as a prediction and optimized tools for experimental studies worldwide. I hope that you will enjoy reading the first issue of the Journal of Modeling and Simulation of Materials. Your valuable feedback will be appreciated for improving the quality of the publication.

January 2018

Dr. Mahmoud Ahmad Editor, Journal of Modeling and Simulation of Materials

\section{How to Cite this Article:}

M. Ahmad, "Importance of Modeling and Simulation of Materials in Research", J. Mod. Sim. Mater., vol. 1, no. 1, pp. 1-2, Jan. 2018. Doi: 10.21467/jmsm.1.1.1-2

\section{References}

[1] N. Bisai and P. Kaw, "Influence of hot and cold neutrals on scrape-off layer tokamak plasma turbulence," Physics of Plasmas, vol. 25, p. 012503, 2018.

[2] S. Meher, M. Carroll, T. Pollock, and L. Carroll, "Designing nickel base alloys for microstructural stability through low $\gamma-\gamma^{\prime}$ interfacial energy and lattice misfit," Materials \& Design, vol. 140, pp. 249-256, 2018.

[3] L. O. Olasunkanmi, B. P. Moloto, I. B. Obot, and E. E. Ebenso, "Anticorrosion studies of some hydantoin derivatives for mild steel in $0.5 \mathrm{M} \mathrm{HCl}$ solution: Experimental, quantum chemical, Monte Carlo simulations and QSAR studies," Journal of Molecular Liquids, vol. 252, pp. 62-74, 2018.

[4] A. Barzegar, C. Pattison, W. Wang, and H. G. Katzgraber, "Optimization of population annealing Monte Carlo for large-scale spinglass simulations," arXiv preprint arXiv:1710.09025, 2017.

[5] A. Möller and V. S. Gullapalli, "System cost and efficiency optimization by heat exchanger performance simulations," Energy Procedia, vol. 129, pp. 459-465, 2017.

[6] K. Matouš, M. G. Geers, V. G. Kouznetsova, and A. Gillman, "A review of predictive nonlinear theories for multiscale modeling of heterogeneous materials," Journal of Computational Physics, vol. 330, pp. 192-220, 2017.

[7] S. Vijay, A. Tugirumubano, S. H. Go, L. K. Kwac, and H. G. Kim, "Numerical simulation and experimental validation of electromagnetic properties for Al-MWCNT-Fe2O3 hybrid nano-composites," Journal of Alloys and Compounds, vol. 731, pp. 465470, 2018.

[8] P. Joshi, J. Singh, R. Sharma, V. Jain, and J. Akhtar, "A facile approach to fabricate ZnO thin film based micro-cantilevers," Microelectronic Engineering, vol. 187, pp. 50-57, 2018.

[9] M. g. Kim, H. Alrowais, and O. Brand, "3D-Integrated and Multifunctional All-Soft Physical Microsystems Based on Liquid Metal for Electronic Skin Applications," Advanced Electronic Materials, 2018.

[10] J. Lawrence, A. Ostendorf, A. Neumeister, S. Dudziak, S. Passinger, and J. Stampfl, "Micro-and Nano-Parts Generated by LaserBased Solid Freeform Fabrication," in Advances in Laser Materials Processing (Second Edition), ed: Elsevier, 2018, pp. 595-633.

[11] H. F. Tibbals, Medical nanotechnology and nanomedicine: CRC Press, 2017.

[12] A. Bahishti, "Peer Review : Critical Process of a Scholarly Publication", Journal of Modern Materials, vol. 2, no. 1, pp. 1.1-1.2, Oct. 2016. 